\title{
Pediatric Tubercular Monoarthritis: A Rare Manifestation and a Case Report
}

\author{
Kaustubh Mohite ${ }^{1}$, Mahesh Mohite ${ }^{2}$, Rachna S Shanbhag ${ }^{3}$
}

\begin{abstract}
Tuberculosis (TB) has always been a burden in developing nations like India. Both pulmonary and extrapulmonary manifestations of TB have been seen widely in the pediatric age group with the specific age-wise distribution. We hereby report a case of a 15-month-old male child who was brought with complaints of localized pain in the left knee and was initially diagnosed to have juvenile-onset oligoarthritis. As the symptoms progressed and a cystic swelling was observed in the left popliteal fossa, he was investigated and aspiration of the cyst was done. Mantoux test done in the child was strongly positive and pus aspirated from the cyst was positive on cartridge-based nucleic acid amplification testing (CBNAAT) for Mycobacterium tuberculosis with rifampicin sensitivity. He was promptly started on 4 drug anti-TB therapy. The pus grew acid-fast bacilli in a culture medium which confirmed the diagnosis. Very young age (15 months) of presentation with cystic lesion from a joint is an unusual presentation of tubercular arthritis.
\end{abstract}

Keywords: Extrapulmonary manifestations, Pediatric, Tuberculosis.

Pediatric Infectious Disease (2021): 10.5005/jp-journals-10081-1303

\section{INTRODUCTION}

Tuberculosis (TB) has proved to have a significant impact on all age groups mainly in the developing nations of the world. Despite the emergence of various newer antitubercular drugs, it has been found out that approximately one-third of the total world's population are said to be silent carriers. According to the WHO report of 2015, a total of 10.4 million new cases of TB were diagnosed worldwide out of which around 1 million were of the pediatric age group., ${ }^{1,2}$ Extrapulmonary TB accounts for up to one-third of all cases. ${ }^{3,4}$ Pediatric age group have a higher predilection to an extrapulmonary manifestation of TB as compared to adults. This is accompanied by higher morbidity and mortality rates. ${ }^{5}$ The lack of reliable TB diagnostic tests for the pediatric population has become the most challenging hurdle in disease control. Both tuberculin skin test (TST) and interferon-gamma release assay (IGRA) fail to differentiate latent from active TB. ${ }^{6}$

\section{Case Description}

A 15-month-old male child was referred to our hospital with complaints of progressive limping of the left lower limb for the past 2 months which was associated with restriction of knee flexion and pain on movement of the knee joint. For these complaints, he was initially diagnosed in an outside hospital to have juvenile-onset oligoarthritis and was started on oral steroids and non-steroidal anti-inflammatory drugs for the past 1 month. As there was no improvement in complaints, he was referred for further detailed workup. On examination, the child was found to have a $3 \times 3 \mathrm{~cm}$ cystic, mobile, fluctuating mass with no overlying scar or sinus (Figs 1 and 2). Ultrasonography of the mass confirmed the cystic nature of the mass and hence aspiration was planned. Aspiration of the cyst drained approximately $3 \mathrm{~mL}$ of purulent fluid which was sent for cartridge-based nucleic acid amplification testing (CBNAAT) for Mycobacterium tuberculosis along with pus culture in Löwenstein-Jensen medium. Hemogram done showed lymphocytic predominance and CBNAAT report for \begin{tabular}{l}
\hline${ }^{1,2}$ Amrut Medical Foundation, Panvel, Maharashtra, India \\
${ }^{3}$ Department of Pediatrics, Smt. Kashibai Navale Hospital, Pune,
\end{tabular} Maharashtra, India

Corresponding Author: Kaustubh Mohite, Amrut Medical Foundation, Panvel, Maharashtra, India, Phone: +91 9833846687, e-mail: drkaustubhmohite@gmail.com

How to cite this article: Mohite K, Mohite M, Shanbhag RS. Pediatric Tubercular Monoarthritis: A Rare Manifestation and a Case Report. Pediatr Inf Dis 2021;3(3):116-118.

Source of support: Nil

Conflict of interest: None

acid-fast bacilli was positive and the strain grew was rifampicin sensitive. Tuberculin skin test done with 2 TU was strongly positive $(16 \times 17 \mathrm{~mm})$ and chest X-ray done showed right-sided perihilar infiltration (Fig. 3). Gastric lavage was sent which did not grow

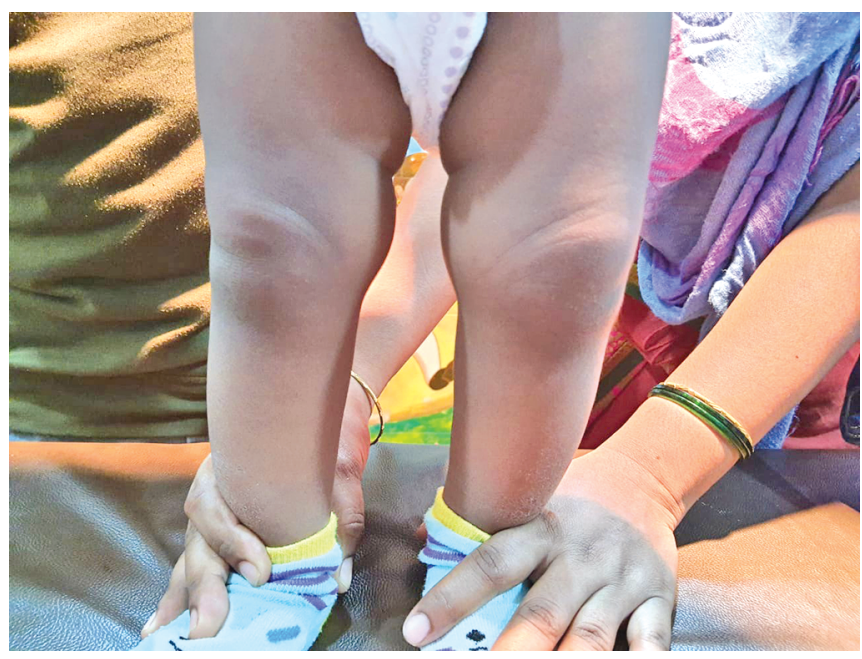

Fig. 1: Swelling of left knee joint

(c) The Author(s). 2021 Open Access This article is distributed under the terms of the Creative Commons Attribution 4.0 International License (https:// creativecommons.org/licenses/by-nc/4.0/), which permits unrestricted use, distribution, and non-commercial reproduction in any medium, provided you give appropriate credit to the original author(s) and the source, provide a link to the Creative Commons license, and indicate if changes were made. The Creative Commons Public Domain Dedication waiver (http://creativecommons.org/publicdomain/zero/1.0/) applies to the data made available in this article, unless otherwise stated. 


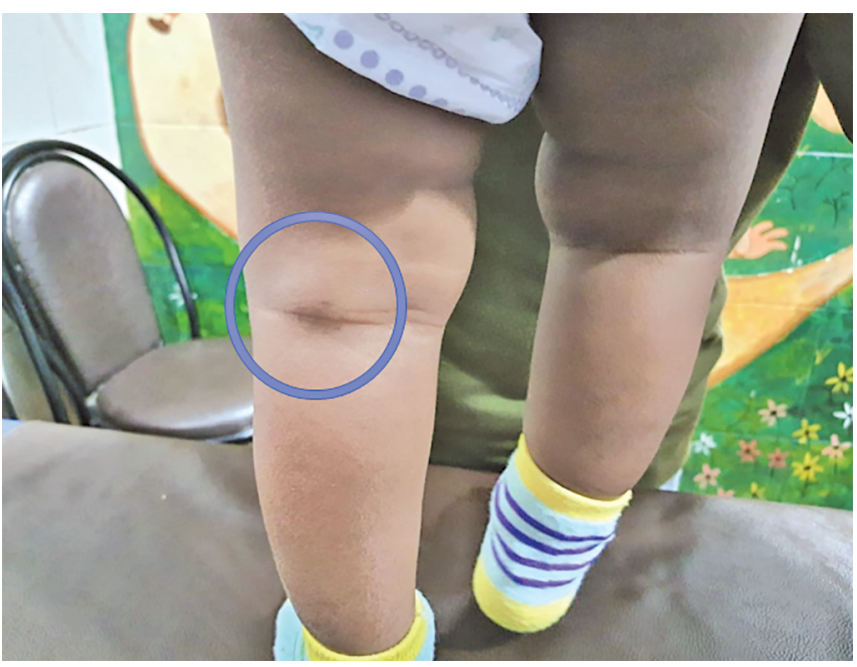

Fig. 2: Cystic mass in the left popliteal fossa

any acid-fast bacilli and was negative for Gene X-pert. The child was immediately started on 4 drug antitubercular drug regimens and was advised follow-up to check for a response. The pus grew acid-fast bacilli in culture medium at the end of 4 weeks which confirmed the diagnosis. The child responded to AKT treatment. On follow-up after 4, 8, and 12 weeks, the cystic lesion seemed to significantly reduce in size.

\section{Discussion}

Isolated monoarthritis caused by M. tuberculosis (in the absence of clinical pulmonary disease) is not a common presentation in age groups $<2$ years. Chronic monoarthritis is a relatively common pediatric problem, with the differential diagnosis being juvenile rheumatoid arthritis (JRA), trauma, viral arthritis, reactive arthritis, Lyme disease, foreign body synovitis, pigmented villonodular synovitis, malignancies, sarcoidosis, and other chronic infections. ${ }^{7}$ In our case, the child had no pulmonary complaints to start with. The child had received BCG immunization at birth along with all the necessary vaccines according to the government schedule. There was no contact of TB patient or history of any chronic disease in the past.

The main pathogenesis involved in tubercular arthritis, as well as tubercular osteomyelitis, is the re-activation of tubercular bacilli which is embedded in the bone during primary infection. The main reason for these bacilli to be affecting the spine and major large joints may be explained by rich vascular supply to these areas. Infrequently, the bacilli may travel from the lung to distant bones by hematogenous or lymphatic modes. Hematogenous dissemination may occur in immunocompromised patients as in individuals with AIDS or transplant recipients.

Tubercular arthritis is most frequently observed in weightbearing joints as seen in our case. ${ }^{8}$ Arthritis with TB may be a result of direct infection of the joint or "reactive" arthritis secondary to visceral disease (i.e., Poncet's disease). Delayed diagnosis is not uncommon in children $<2$ years of age. The main complaints of TB arthritis include pain, stiffness, effusion, and low-grade fever. The characteristic insidious chronic course of this disease makes it difficult to distinguish from other forms of chronic arthritis. ${ }^{8,9}$ Synovial fluid is typically inflammatory with a total leukocyte count ranging from 10,000 to 20,000 cells $/ \mathrm{mm}^{3}$ and polymorphonuclear cell predominance. Protein in $>3.5 \mathrm{~g} / \mathrm{dL}$ and glucose levels are

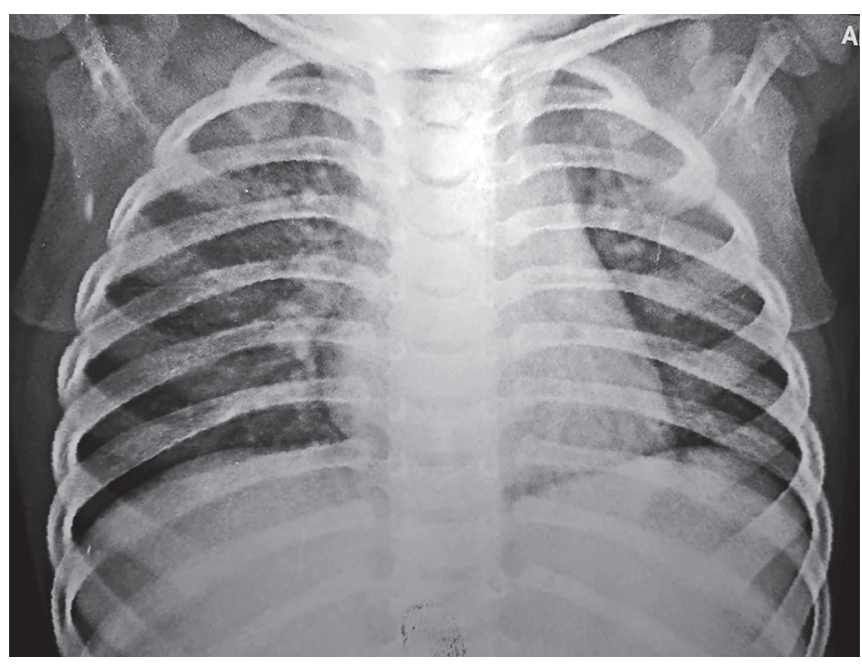

Fig. 3: Chest X-ray-right perihilar infiltration

normal. Radiological findings depend on the stage of arthritis. Early features include soft tissue swelling and periarticular osteopenia which later progresses to the blurring of the subchondral bone surface and marginal erosion with joint narrowing and frank destruction of bone.

Most cases of TB arthritis (including our case) have no pulmonary symptoms.

Tuberculosis monoarthritis is to be considered as a primary case and the treatment schedule should be planned accordingly. The regimen includes 2 months of 4 drug intensive therapy; isoniazid, rifampicin, pyrazinamide, and ethambutol which is to be followed by a continuation phase of 4 months with isoniazid, rifampicin, and ethambutol (according to NTEP 2020). Compliance with medication is of utmost importance in the prevention of medication failure and the emergence of drug resistance.

\section{ConCLUSION}

Pediatric tubercular monoarthritis is a rare entity in the age group $<2$ years. The absence of any other systemic features of TB makes it difficult to come to a final diagnosis and hence we should have a low threshold for diagnosing TB, especially in endemic regions.

\section{INVESTIGATION}

Hemogram: $\mathrm{Hb}-10.6 \mathrm{~g} / \mathrm{dL}$.

TLC - 13,500/ $\mathrm{mm}^{3}$ (Neutrophil-37\%, Lymphocytes-58\%).

Platelet-4.45 lac $/ \mathrm{mm}^{3}$.

ANA (immunofluorescence)-weak positive.

Mantoux test $-16 \times 17 \mathrm{~mm}$.

Gene X-pert-rifampicin sensitive AFB detected.

Rapid TB culture of Pus-AFB seen.

\section{References}

1. Maltezou HC, Spyridis P, Kafetzis DA. Extra-pulmonary tuberculosis in children. Archi Dis Childhood 2000;83(4):342-346. DOI: 10.1136/ adc.83.4.342.

2. Khurana AK, Dhingra $B$. What is new in management of pediatric tuberculosis? Indian Pediatr 2019;56(3):213-220. DOI: 10.1007/s13312019-1503-9.

3. Rieder HL, Snider JrDE, Cauthen GM. Extrapulmonary tuberculosis in the united states 1-3. Am Rev Respir Dis 1990;141(2):347-351. DOI: 10.1164/ajrccm/141.2.347. 
4. Weir MR, Thornton GF. Extrapulmonary tuberculosis. Experience of a community hospital and review of the literature. Am J Med 1985;79(4):467-478. DOI: 10.1016/0002-9343(85)90034-8.

5. Starke JR. Resurgence of tuberculosis in children. Pediat Pulmonol 1995;19(S11):16-17. DOI: 10.1002/ppul.1950191110.

6. Sulis G, Roggi A, Matteelli A, et al. Tuberculosis: epidemiology and control. Mediterran J Hematol Infect Dis 2014;6(1):e2014070. DOI: 10.4084/mjhid.2014.070.
7. Al-Matar MJ, Cabral DA, Petty RE. Isolated tuberculous monoarthritis mimicking oligoarticular juvenile rheumatoid arthritis. J Rheumato 2001;28(1):204-206.

8. Carender CN, Akoh CC, Kowalski HR. Mycobacterium tuberculosis monoarthritis of the knee in children: a case report. lowa Orthop J 2018;38:17.

9. Veloci S, Mencarini J, Lagi F, et al. Tubercular prosthetic joint infection: two case reports and literature review. Infection 2018;46(1):55-68. DOI: 10.1007/s15010-017-1085-1. 